2020, with the main strategy being treatment of entire endemic communities. Since the inception of the Global Programme for the Elimination of LF in 2000, tremendous progress has been made in many endemic countries. However, current observations point to the need for improved treatment regimen, frequency of treatment or drug delivery strategies in order to achieve the elimination goals in certain endemic areas. In this randomised trial, we evaluate the use of twiceyearly treatment with ivermectin and albendazole in 18 LFendemic communities in Ghana, where despite 15 years of yearly treatment the disease is still above the elimination thresholds.

Methods Following demographic data collection, Wuchereria bancrofti antigen, microfilaria and antibody prevalence were assessed in study participants using the Alere FTS kit, nucleopore filtration and Wb123 ELISA, respectively. The study assessed the perspectives of the communities'on persistent transmission of LF in view of implementing effective treatment uptake strategies.

Results The baseline assessments revealed antigen prevalence of $8.2 \%(95 \% \mathrm{CI}=6.8-9.8)$, with overall microfilaria prevalence of $1.2 \%$. Infections were higher in males and in individuals who spend significant amount of time outdoors for commercial activities. Barriers related to medication, personal, health system, disease and social structure were observed to affect mass drug administration compliance. Community members perceived that they were not susceptible to infection and this together with drug adverse effects strongly affect the ingestion of the drugs.

Conclusion While this trial is still in an early phase, the baseline assessments reveal programmatic challenges to the implementation of a twice-yearly treatment strategy for the control of LF which must be addressed to enhance implementation success.

\section{PO 8248 DETERMINANTS OF ACCEPTABILITY OF MALARIA RAPID DIAGNOSTIC TEST AMONG HEALTH WORKERS IN KINTAMPO NORTH MUNICIPALITY, GHANA}

${ }^{1}$ Michael Anaba*, ${ }^{1}$ Kenneth Ae-Ngebisi, ${ }^{2}$ Seth Owusu Agyei, ${ }^{3}$ Latifat Ibisomi. ${ }^{1}$ Kintampo Health Research Center, Kintampo, Ghana; ${ }^{2}$ University of Health and Allied Sciences, Hohoe, Ghana; ${ }^{3}$ University of the Witwatersrand, Johannesburg, South Africa

\subsection{6/bmjgh-2019-EDC.58}

Background Ghana rolled out the policy in 2013 with the use of malaria rapid diagnostic test (mRDT) promoted to facilitate diagnosis. However, health workers who are at the centre of mRDT implementation still treat half of febrile patients with negative mRDT results with antimalarial drugs, suggesting limited or lack of acceptability of the innovation.

Methods We conducted a cross-sectional study to examine determinants of mRDT among health workers in Kintampo North Municipality (KNM) in Ghana. Data were collected from 110 health workers in KNM involved in malaria management from February to April 2017. The survey tool was based on two frameworks - the Technology Acceptance Model (TAM) and Normalisation Process Theory (NPT). Acceptability was measured by ease of use, perceived usefulness and intention to use.

We hypothesised that acceptability was influenced by coherence, cognitive participation, collective action, reflexive monitoring and respondent characteristics. A composite acceptability score was computed from a 21-item questionnaire for each respondent. The respondents were divided into three groups of low, moderate and high acceptability for ordered logistic regression to examine the relationship between acceptability and its determinants.

Results The median acceptability score was 84(Q1, Q3:68, 103). About $34 \%$ of health workers had low acceptability while $37 \%$ and $29 \%$ had moderate and high acceptability respectively. In the multivariable analysis, coherence $(\mathrm{OR}=1.23, \quad 95 \% \mathrm{CI}=1.11-1.37), \quad$ cognitive participation $(\mathrm{OR}=1.35,95 \% \mathrm{CI}=1.10-1.66)$, health workers in rural health facilities $(\mathrm{OR}=6.99,95 \% \mathrm{CI}=1.82-26.84)$ and health workers with more than three years' experience $(\mathrm{OR}=5.53,95 \%$ $\mathrm{CI}=1.98-15.42$ ) were more likely to have high mRDT acceptability.

Conclusion Acceptability of mRDT was moderate among the majority. This can be improved by enhancing health workers' coherence on the benefits of mRDT through policy building or dissemination of information, promoting health workers' cognitive participation in the mRDT implementation process through recruitment of local 'champions' to promote 'buy-in' and providing incentives to health workers to embed and sustain the use of the health technology.

\section{PO 8249 SUBMICROSCOPIC PLASMODIUM FALCIPARUM INFECTIONS IN MATCHED PERIPHERAL, PLACENTAL AND UMBILICAL CORD BLOOD SAMPLES FROM CONGOLESE WOMEN AT DELIVERY}

${ }^{1}$ Yvon Mbouamboua*, ${ }^{1}$ Félix Koukouikila-Koussounda, ${ }^{2} J a c q u e s$ Van Helden, ${ }^{1}$ Francine Ntoumi. ${ }^{1}$ Fondation Congolaise pour la Recherche Médicale, Brazzaville, Republic of the Congo; ${ }^{2}$ Aix-Marseille University, Institute de Neurosciences des Systèmes, Marseille, France

\subsection{6/bmjgh-2019-EDC.59}

Background This cross-sectional study was conducted to characterise $P$. falciparum infections matched in peripheral, placental and cord blood among Congolese women at delivery receiving 1, 2 or more doses of sulfadoxine-pyrimethamine. The cross-sectional study was conducted in a Southern district of Brazzaville, Republic of the Congo, between March 2014 and April 2015.

Methods Peripheral and placental blood samples were collected for P. falciparum infection investigation by microscopy and nested polymerase chain reaction (PCR), using P. falciparum merozoite surface protein-2 (msp2) gene as marker.

Results Of the 370 pregnant women recruited, only $7.3 \%$ peripheral and $2.7 \%$ placental blood samples were found smear-positive for $P$. falciparum by microscopy. All isolates from cord blood were microscopy-negative. However, the prevalences of submicroscopic $P$. falciparum infections (detectable only by PCR) were $25.4 \%, 16.7 \%$ and $9.4 \%$ in peripheral, placental and cord blood respectively. The frequency of 3D7 msp2 alleles was the highest $(>60 \%)$ whatever the blood considered. We found a high prevalence of submicroscopic infection in pregnant women associated with a high genetic diversity of $P$. falciparum isolates. The multiplicity of infection ranged between 1.2 and 1.4 irrespective of the blood compartment, and it showed no significant association with maternal age $(p=0.3)$, gravidity $(p=0.1)$ or sulfadoxinepyrimethamine $(p=0.3)$.

Conclusion In summary, this study showed that there is a high prevalence of submicroscopic infection and a high genetic diversity of Plasmodium falciparum strains in Congo. This 